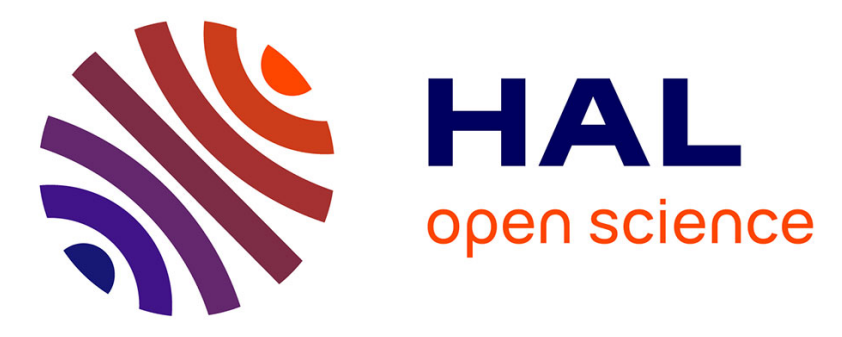

\title{
Determination of the electron anomalous mobility through measurements of turbulent magnetic field in Hall thrusters
}

\author{
A. Lazurenko, Thierry Dudok de Wit, Claude Cavoit, Vladimir \\ Krasnoselskikh, André Bouchoule, Michel Dudeck
}

\section{To cite this version:}

A. Lazurenko, Thierry Dudok de Wit, Claude Cavoit, Vladimir Krasnoselskikh, André Bouchoule, et al. Determination of the electron anomalous mobility through measurements of turbulent magnetic field in Hall thrusters. Physics of Plasmas, 2007, 14 (3), pp.033504. 10.1063/1.2535813 . insu02932979

\section{HAL Id: insu-02932979 \\ https://hal-insu.archives-ouvertes.fr/insu-02932979}

Submitted on 8 Sep 2020

HAL is a multi-disciplinary open access archive for the deposit and dissemination of scientific research documents, whether they are published or not. The documents may come from teaching and research institutions in France or abroad, or from public or private research centers.
L'archive ouverte pluridisciplinaire HAL, est destinée au dépôt et à la diffusion de documents scientifiques de niveau recherche, publiés ou non, émanant des établissements d'enseignement et de recherche français ou étrangers, des laboratoires publics ou privés. 


\section{Determination of the electron anomalous mobility through measurements of turbulent magnetic field in Hall thrusters}

Cite as: Phys. Plasmas 14, 033504 (2007); https://doi.org/10.1063/1.2535813

Submitted: 06 October 2006 . Accepted: 17 January 2007 . Published Online: 05 March 2007

A. Lazurenko, T. Dudok de Wit, C. Cavoit, V. Krasnoselskikh, A. Bouchoule, and M. Dudeck

\section{ARTICLES YOU MAY BE INTERESTED IN}

Tutorial: Physics and modeling of Hall thrusters

Journal of Applied Physics 121, 011101 (2017); https://doi.org/10.1063/1.4972269

Magnetic shielding of a laboratory Hall thruster. II. Experiments

Journal of Applied Physics 115, 043304 (2014); https://doi.org/10.1063/1.4862314

Magnetic shielding of a laboratory Hall thruster. I. Theory and validation

Journal of Applied Physics 115, 043303 (2014); https://doi.org/10.1063/1.4862313

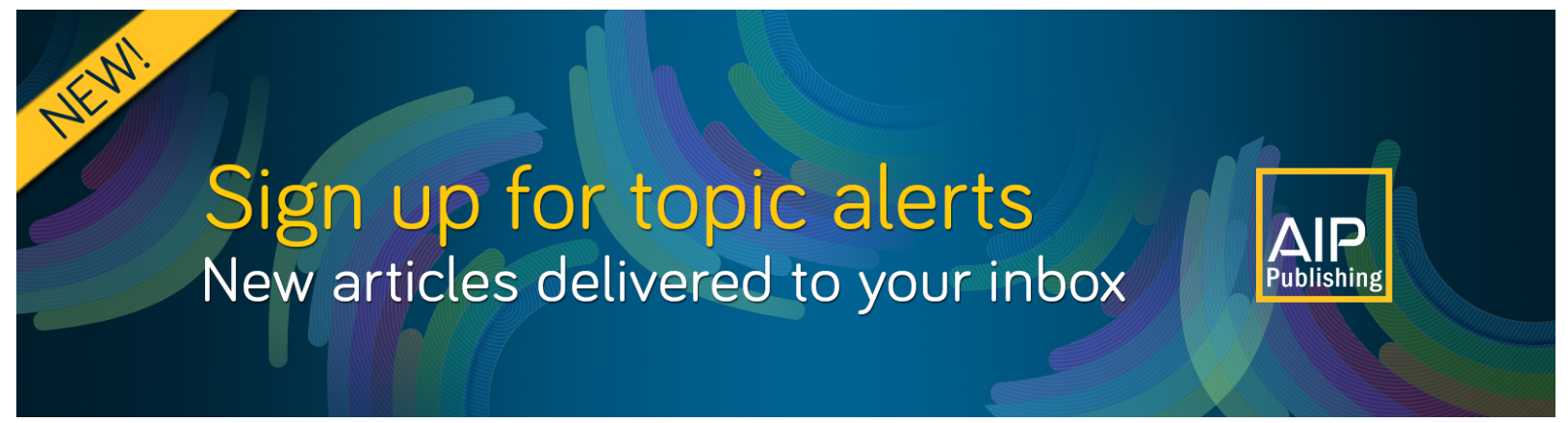




\title{
Determination of the electron anomalous mobility through measurements of turbulent magnetic field in Hall thrusters
}

\author{
A. Lazurenko ${ }^{a)}$ \\ ICARE, CNRS, 45071 Orléans, France
}

T. Dudok de Wit, C. Cavoit, and V. Krasnoselskikh

LPCE, CNRS and Orléans University, 45071 Orléans, France

A. Bouchoule
GREMI Laboratory, CNRS and Orléans University, 45067 Orléans, France

M. Dudeck

ICARE, CNRS, 45071 Orléans, France

(Received 6 October 2006; accepted 17 January 2007; published online 5 March 2007)

\begin{abstract}
Measurements of the turbulent magnetic field in a Hall thruster have been carried out between $1 \mathrm{kHz}$ and $30 \mathrm{MHz}$ with the aim of understanding electron transport through the magnetic field. Small detecting coils at the exit of the accelerating channel and outside of the ionic plume were used to characterize various instabilities. The characteristic frequencies of the observed power spectral densities correspond to known classes of instabilities: low frequency $(20-40 \mathrm{kHz})$, transit time $(100-500 \mathrm{kHz})$, and high frequency $(5-10 \mathrm{MHz})$. A model of the localized electron currents through a magnetic barrier is proposed for the high-frequency instability, and is found to be in good quantitative agreement with the observations. Based on the measured high-frequency turbulent magnetic field, the turbulent electric field is estimated to be about $1 \mathrm{~V} / \mathrm{cm}$ outside of the plume and ranges from 10 to $10^{2} \mathrm{~V} / \mathrm{cm}$ at the channel midradius at the exit of the thruster. The "anomalous" electron collision frequency, related to the high-frequency instability, is estimated to be $<10^{6} \mathrm{~s}^{-1}$, which largely exceeds the classical frequency in the core of the exit plasma but is lower than the frequency that is generally used in hybrid codes. (c) 2007 American Institute of Physics.
\end{abstract}

[DOI: $10.1063 / 1.2535813$ ]

\section{INTRODUCTION}

The Hall thruster is a space propulsion technology that was initially developed in the 1970s in the frame of the Soviet space program ${ }^{1,2}$ and which is frequently used today by geostationary satellites for North-South and East-West station-keeping (see, for example, Ref. 3). Recently, this type of thruster was implemented as the main propulsion on the interplanetary mission "SMART-1.",

The Hall thruster is a plasma device that is based on the so-called ion accelerator with closed electron drift. A mostly radial magnetic field $B$ is created across an annular ceramic channel, with a maximum intensity near the channel exit. The value of magnetic field is chosen in such a way that the electron Larmor radius is much smaller than the channel dimensions, whereas the ion Larmor radius is much larger. The electrons are therefore magnetized but the magnetic field does not affect ion motions. The dc discharge voltage $U_{d}$ is applied between an anode, located at the bottom of the channel, and an external hollow cathode. A propellant (xenon) is injected through the anode structure. The reduction of longitudinal mobility of electrons across the radial magnetic field leads to a localized axial voltage drop of the order of the

\footnotetext{
${ }^{a)}$ Electronic mail: Alexey.Lazurenko@cnrs-orleans.fr
}

anode-cathode discharge voltage. In such an "E cross B" configuration, electrons drift in the azimuthal direction with an average azimuthal velocity $E_{z} / B_{r}$. Typical values of $E_{z}\left(10^{4} \mathrm{~V} / \mathrm{m}\right)$ and $B_{r}(20 \mathrm{mT})$ lead to average electron energies higher than $10 \mathrm{eV}$ and this magnetically confined electron cloud is very efficient for ionizing the propellant gas. The ions are accelerated toward the exit by the electric field, leaving the channel with kinetic energies of the order of the discharge potential. The thrust corresponds to the momentum delivered to this escaping ion flow. A detailed description of Hall thrusters can be found in Refs. 1, 2, and 5.

One of the key questions of Hall thruster physics is the understanding of electron transport through the magnetic field. Classical diffusion approaches fail to give a satisfactory agreement with the experiment. A correct description of the electron transport, however, is very important for making numerical codes progress toward fully predictive codes. Hybrid-type codes, for example, where electrons are described as a fluid and ions as particles, use artificial viscosity and the diffusion coefficients are chosen to properly describe macroscopic plasma motions. ${ }^{6-11}$ The determination of these coefficients is a crucial problem for these simulations. Two hypotheses are generally proposed to explain this "anomalous" electron transport: the so-called "near-wall" conductivity, where the electron collisions with walls increase the effective collision frequency, and an oscillation-assisted (or 
turbulent) electron transport. ${ }^{1,2,12}$ While inside the accelerating channel these two mechanisms can compete with each other, outside only the latter can exist.

One of the approaches for determining the electron transport coefficients experimentally is to measure the local time-averaged plasma properties and to deduce the electron mobility from the electron current equation. ${ }^{13,14}$ Another way consists in studying the properties of the plasma oscillations and deducing the "effective" electron collision frequency from the analysis of the oscillation intensities. ${ }^{13,15,16}$ While the first approach does not differentiate between the two anomalous electron transport mechanisms, the second one informs only on the turbulent transport. Meanwhile, the second approach provides insight into the way turbulent transport works.

The gas discharge in Hall thrusters generates a number of plasma instabilities whose intensities depend strongly on the thruster operating parameters, notably the discharge voltage and the magnetic field. ${ }^{1,2,12}$ Numerous techniques have been implemented to study experimentally the physics of Hall thrusters, and in particular plasma instabilities. Ex situ diagnostics include the observation of instability signatures in the electric power circuit, or the space-averaged detection of light emission from the channel and plume. In situ diagnostics, in the thruster channel and in the thruster plume, include electric probes, arrays of optical fibers, the implementation of time-of-flight techniques and special discharge interrupters, or inductive coils. A comprehensive review of physical insight obtained with these different techniques is given in Ref. 17.

The detection of magnetic fields generated by charged particle currents in the Hall thruster plasma provides important insight into thruster physics, and especially into nonstationary processes. A wire loop around the channel circumference was used in a number of previous investigations to record the variation of magnetic field from the electron azimuthal drift current and to investigate its spatial and temporal evolution. ${ }^{17-20}$ Magnetic probes are widely used in different laboratory plasma devices, in particular in magnetized plasma accelerators (see, for example, Ref. 21).

In this paper, we present an implementation of the magnetic field detection technique for studying plasma instabilities in Hall thrusters. This diagnostic is based on the localized (in azimuth, axial, and radial directions) detection of the time-varying magnetic field generated in the plasma volume. The driving interest is to shed light on the generation mechanism of instabilities in the $5-10 \mathrm{MHz}$ frequency range, ${ }^{2,12,22-27}$ and to evaluate from this the diffusion coefficient that would be needed for hybrid codes. Meanwhile, we found out that this technique is also suitable for studying different types of instabilities in the wide frequency range.

This paper is organized as follows. In Sec. II, a description of the experimental setup is given. In Sec. III, the experimental results are presented, which are followed by a more detailed interpretation in Sec. IV. The conclusions are formulated in Sec. V.

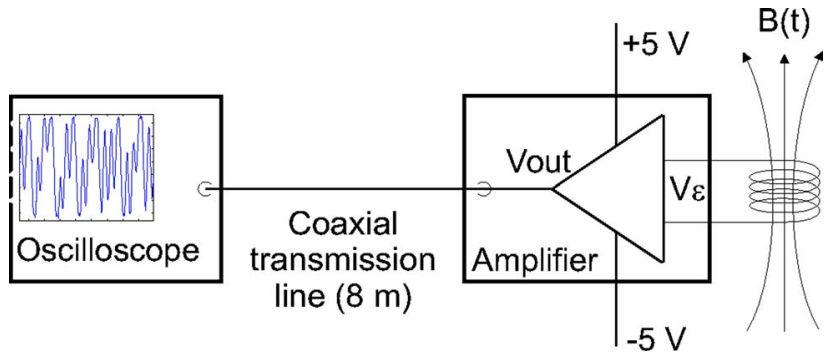

FIG. 1. (Color online) Configuration of the measurement system.

\section{EXPERIMENTAL SETUP}

\section{A. Principle of diagnostics}

A nonstationary magnetic field generates a voltage drop in a coil positioned in such a way that the force lines cross the coil surface. The underlying effect is described by Faraday's law,

$$
\varepsilon=-\frac{d \Phi}{d t}, \quad \Phi=N S B,
$$

where $\varepsilon$ is the electromotive force (voltage generated in the coil), $\Phi$ is the magnetic flux crossing the coil surface, $N$ is the number of turns in the coil, $S$ is the coil surface, and $B$ is the magnetic induction. With a properly oriented coil axis, it is possible to detect selectively the variations of any component of a nonstationary magnetic field. Such a coil has a linear frequency response. Indeed, for a magnetic field that is generated by the current flowing in an infinitely long straight wire,

$$
B=\mu \mu_{0} \frac{I}{2 \pi r},
$$

where $\mu$ is the relative magnetic permeability, $\mu_{0}=4 \pi$ $\times 10^{-7} \mathrm{H} / \mathrm{m}, I$ is the intensity of the current in the wire, and $r$ is the distance from the wire. With the ansatz $I=I_{0} e^{-j \omega t}$, then

$$
\varepsilon=-\frac{d \Phi}{d t}=j \mu \mu_{0} \frac{N S}{2 \pi r} \omega I=j \mu \mu_{0} f \frac{N S}{r} I,
$$

and $\varepsilon$ is therefore a linear function of the frequency $f$. Such coils are routinely used for investigating ionospheric plasmas. $^{28,29}$

\section{B. Configuration of the measurement system}

The detecting coil diameter was chosen a priori to be equal to $9 \mathrm{~mm}$, resulting from the tradeoff between a compact size and easiness in fabrication and handling. To define the shape of the measurement system, let us assume that the typical discharge current of $4.2 \mathrm{~A}$ is concentrated on the thruster axis and oscillates at the $20 \mathrm{kHz}$ frequency of the bulk instability. ${ }^{1,2,12}$ Then, according to Eq. (3), the magnetic field from this current induces the voltage drop of $\sim 100 \mu \mathrm{V}$ in the $9 \mathrm{~mm}$ coil of one turn, located at $r=50 \mathrm{~mm}$ from the thruster axis. Each coil should therefore have several turns to increase the signal level and should be closely followed by an amplifier to avoid signal degradation (Fig. 1). Such a 


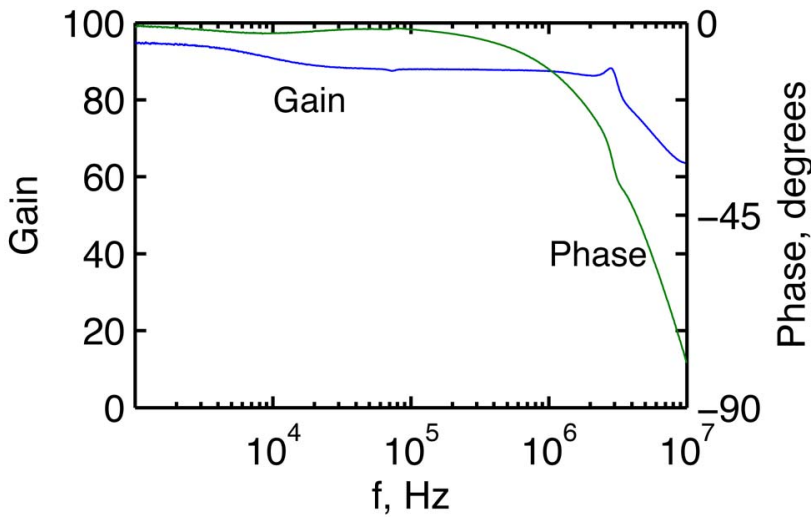

FIG. 2. (Color online) Amplifier characteristics.

design is inherited from the instrumentation for ionospheric investigations. $^{29}$

The coils are built from ordinary $0.8 \mathrm{~mm}$ enameled transformer wire. Each coil has 18 turns $9 \mathrm{~mm}$ in diameter with overall longitudinal dimension of $7 \mathrm{~mm}$. The length of the coil's free ends is $80 \mathrm{~mm}$. The measured self-inductance of the coils is on the order of $2.3 \mu \mathrm{H}$ at $10 \mathrm{kHz}$.

The amplifiers are mounted on a printed circuit board (PCB), with two amplifiers on each PCB (one amplifier for each coil). The $50 \Omega$ output impedance of the amplifiers is adapted to the transmission lines, using a SMA connector to connect the cables to the PCBs. The signals are transferred through $8 \mathrm{~m}$ coaxial lines and are observed and recorded on the digital four-channel Tektronix 5104B oscilloscope.

\section{Calibration of coils and amplifiers}

The amplifiers were first calibrated separately from the coils by applying on the input a sinusoidal voltage of variable frequency in the $10^{3}-10^{7} \mathrm{~Hz}$ range. The amplifier gain is quite flat (90-100) up to $10^{6} \mathrm{~Hz}$, whereas for high frequencies of $10^{7} \mathrm{~Hz}$ it drops to $\sim 60$ (Fig. 2). The amplifiers also shift the phase at high frequencies (see Fig. 2).

The coils and the amplifiers were then calibrated together according to the following scheme (Fig. 3): a long metallic bar was inserted into a circuit carrying a sinusoidal current of variable frequency. The coils were placed at $50 \mathrm{~mm}$ from the bar with the surface of the coil perpendicular to the magnetic field. The amplifier output voltage $U_{\text {acq }}$ was compared to the input voltage $U_{\text {gen }}$ to get the transfer function of the system. The resulting Bode plot is presented in Fig. 4. Notice a resonance at $30 \mathrm{MHz}$.

The gain of the system "coil+amplifier" can be represented as

$$
\text { Gain }=\operatorname{Gain}(\omega)=\frac{U_{\mathrm{acq}}}{U_{\mathrm{gen}}}=\frac{U_{\mathrm{acq}}}{I_{\mathrm{gen}} R_{50 \Omega}},
$$

where $\omega$ is the pulsation. Taking into account Eq. (2) for the magnetic field of a long wire, one can write the magnetic field as

$$
B(\omega)=U_{\mathrm{acq}} f_{\mathrm{tr}}(\omega),
$$

where $f_{\text {tr }}(\omega)$ is the "effective" transfer function,

\section{Oscilloscope - generator}

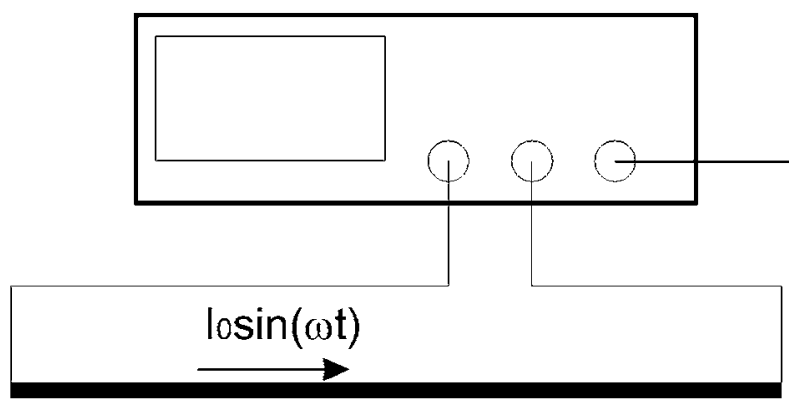

Metallic bar

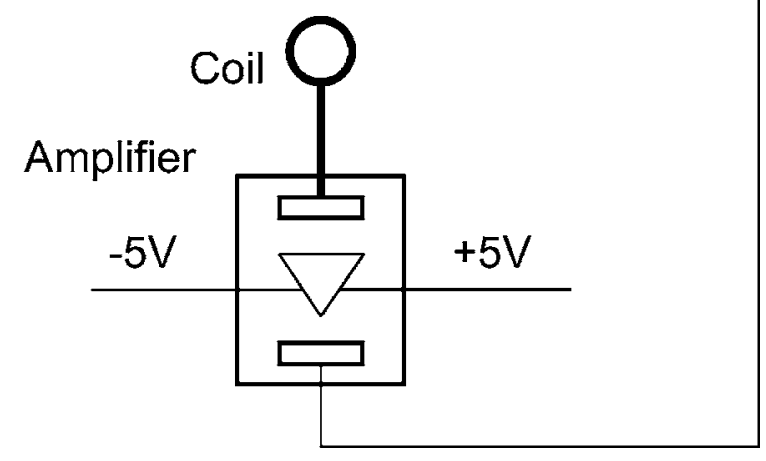

FIG. 3. Scheme of the amplifier and coil calibration setup.

$$
f_{\mathrm{tr}}(\omega)=\frac{1}{2 \pi \mu \mu_{0} d R_{50 \Omega} \mathrm{Gain}} .
$$

\section{Installation on the thruster}

The measurements were carried out on the Hall thruster SPT-100ML at the French national facility PIVOINE. ${ }^{30}$ The thruster operated at its nominal operation mode, with a massflow rate of $4.5 \mathrm{mg} / \mathrm{s}$, a discharge voltage of $U_{d}=300 \mathrm{~V}$, and a discharge current of $I_{d}=4.2 \mathrm{~A}$.

Three coils were installed at the thruster exit in front of the external magnetic pole around the channel (Fig. 5) on a radius $70 \mathrm{~mm}$. The axes of two coils (B7 and B3 on Fig. 5) were oriented azimuthally, and the last coil B6 was oriented

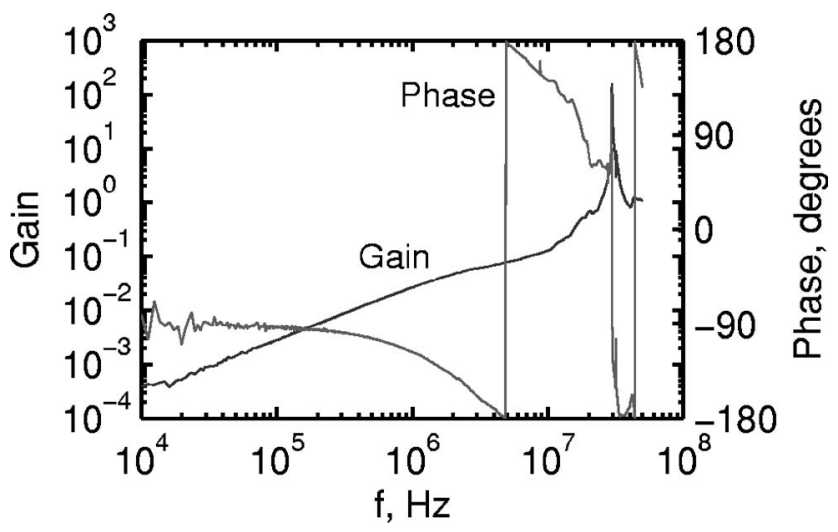

FIG. 4. Frequency characteristics of the "coil+amplifier" system. This system has a resonance at $30 \mathrm{MHz}$. 


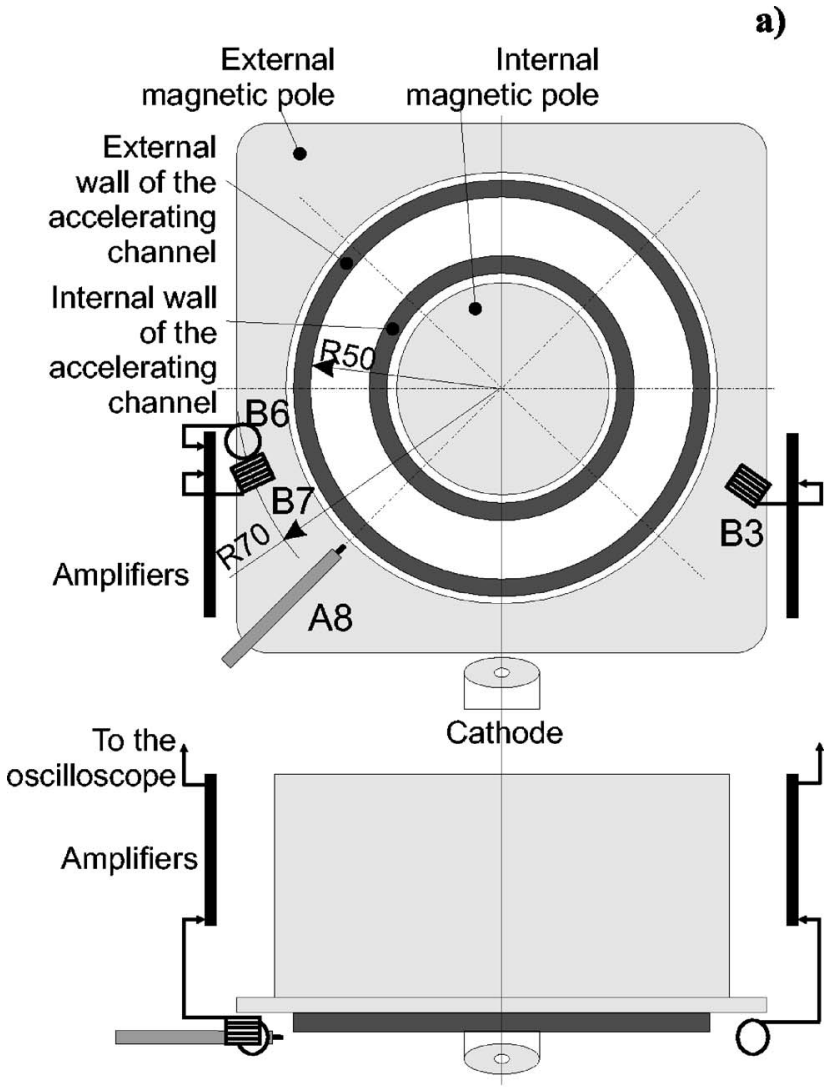

b )

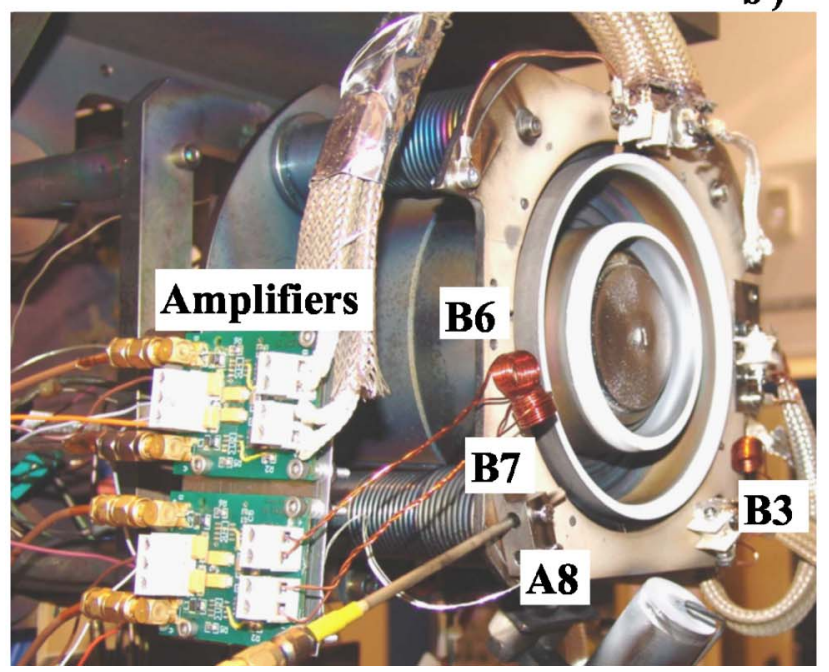

FIG. 5. (Color online) Location of the magnetic field coils and the Langmuir probe on the SPT-100ML thruster. (a) Schematics; the B6 coil is oriented axially, while the B7 and B3 are oriented azimuthally. A8 stands for the coaxial Langmuir probe. (b) Picture of the SPT-100ML thruster, with the coils and the probe. Some additional diagnostics are seen that are not pertinent for this study.

with its axis parallel to the thruster axis. Therefore, B7 and B3 detect the azimuthal component of the fluctuating magnetic field, whereas coil $\mathrm{B} 6$ detects the axial component. A shielded Langmuir probe ${ }^{27}$ was also installed at the same exit cross section of the thruster, and it will be referred to as A8 (see Fig. 5). The probe operated at practically ground potential; the signal from the probe was directly observed on the oscilloscope at $50 \Omega$ ac operation mode.
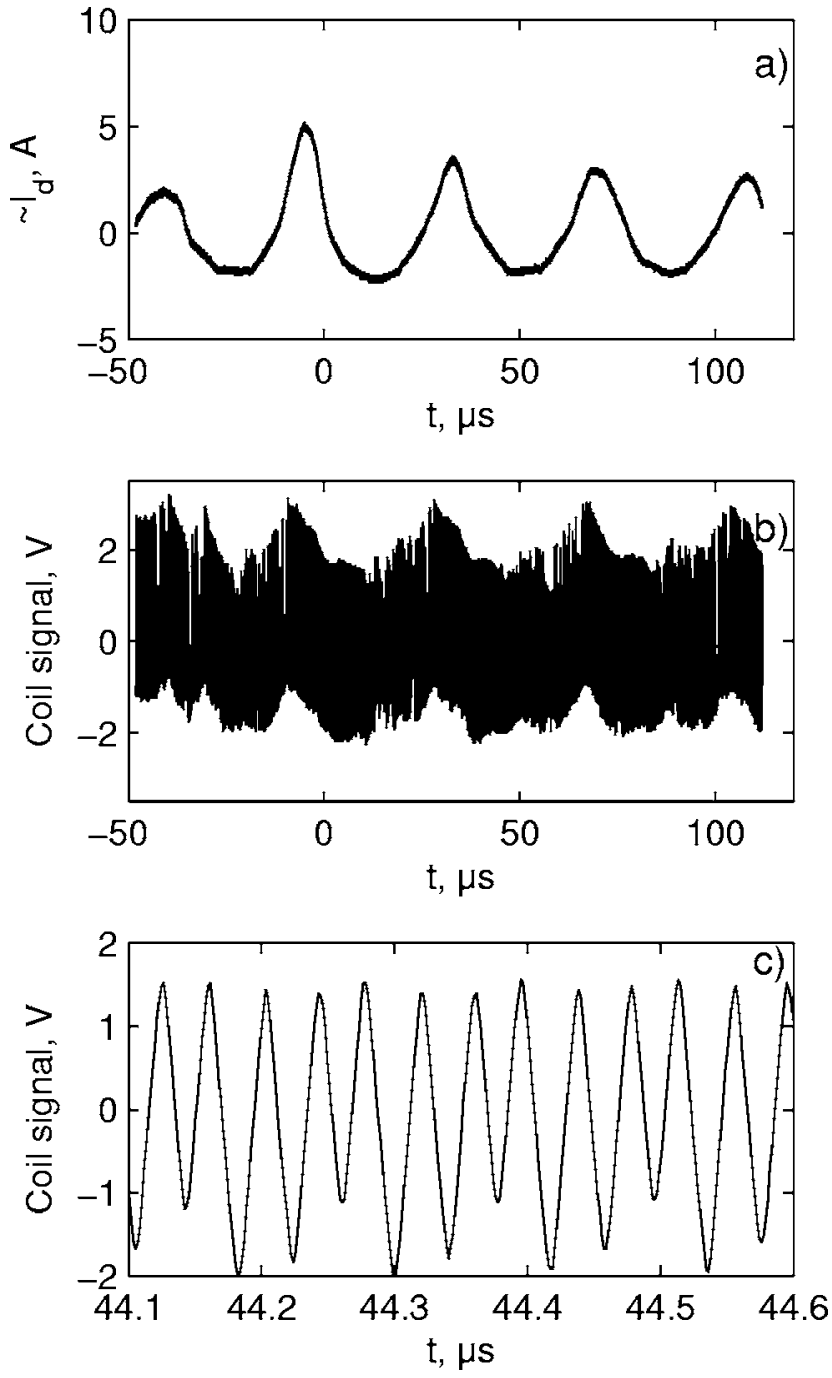

FIG. 6. Time evolution of the discharge current (a), a typical coil B7 signal (b), and an excerpt of the same coil signal (c). Notice that the coil signal has the same LF variations as the discharge current.

The amplifiers were installed in the lateral zones of the thruster in order to be close enough to the coils $(80 \mathrm{~mm})$ while being protected from the excessive heat flux.

Oscillations in discharge current were also recorded using Tektronix P6022 current probes installed on the power circuit.

\section{RESULTS}

\section{A. Coil signals}

A typical coil recording is presented in Fig. 6 along with the wave form of the discharge current. One can directly observe the modulation of the coil signal at the frequency of discharge current oscillations at $25 \mathrm{kHz}$. There are also some intervals where the signal level strongly drops, which we attribute to the instantaneous saturation of the amplifier. Such regimes are excluded from our analysis.

We calculated the power spectral density of the raw signals separately for two frequency bands: 

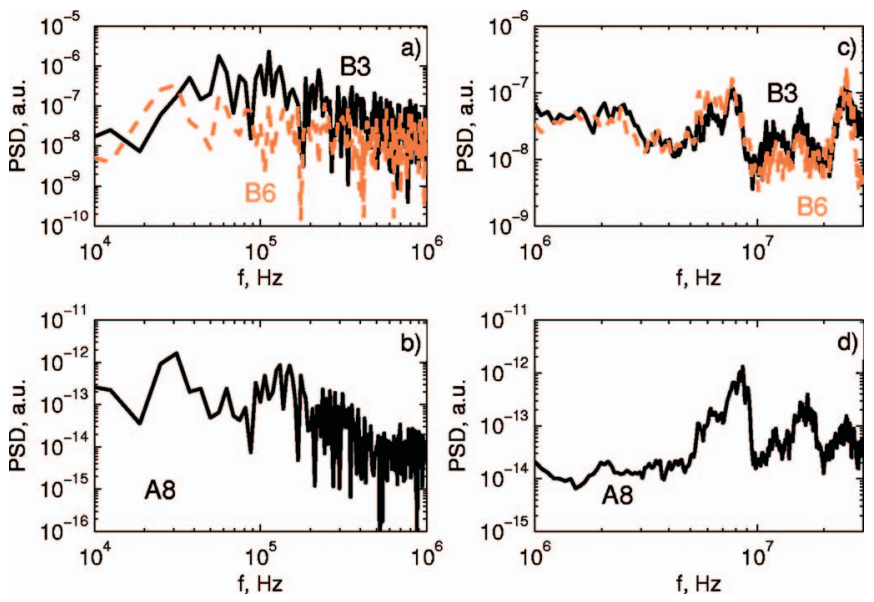

FIG. 7. (Color) Power spectral densities of the coil signals and of the probe signal. Left panels for $f<1 \mathrm{MHz}$ and right panels for $f>1 \mathrm{MHz}$. Logarithmic scales are used for the axes. (a) shows low-frequency spectra from coil B3 (black) and B6 (red dashed). Notice a relative depletion of the spectrum of B6 in the 100-500 kHz range. (b) shows the low-frequency spectrum of the A8 probe. (c) shows high-frequency spectra of coils B3 (black) and B6 (red dashed), with similar spectra. (d) shows the high-frequency spectrum of the A8 probe.

(i) $\quad$ For $f<1 \mathrm{MHz}$, using Welch's periodogram method with a single Hamming window.

(ii) For $f>1 \mathrm{MHz}$ using the same method, with a sliding Hamming window of $2^{13}$ samples and 50\% overlapping.

Typical spectra are represented in Fig. 7 for the coils B3, B6 and for the Langmuir probe A8. The spectrum of the B7 coil is close to that of the B3 one and is not shown. Three characteristic frequency bands can be distinguished in the spectra: $20-40 \mathrm{kHz}, 100-500 \mathrm{kHz}$, and $8-24 \mathrm{MHz}$.

The $20-40 \mathrm{kHz}$ frequency range corresponds to the lowfrequency (LF) oscillations in Hall thrusters, often referred to as "contour" or bulk oscillations. ${ }^{1,2,12,31}$ These oscillations are conventionally considered as being the most important ones and are associated with the displacement of the ionization front; ${ }^{32}$ they are synchronous in the whole plasma volume. The modulation of the discharge parameters at these frequencies can reach up to $100 \%$; usually, the values are significantly lower by adjustment of the magnetic field, and therefore do not affect the operation of the thruster. ${ }^{2}$ In our experiment, the orientations of the coils suggest that different physical sources contribute to the coil signals of B3 and B7 on the one hand and to B6 on the other hand. Coils B3 and B7 detect the azimuthal magnetic field that is generated by currents of charged particles having axial and radial components. These currents are carried by ions leaving the thruster, which have significant axial and radial components and negligible azimuthal component, and corresponding components of electron current. These currents are modulated at a LF scale. Coil B6 detects the axial magnetic field that is generated by azimuthal and radial currents. Such currents are made out of the radial components of ion and electron currents and the azimuthal component of electron current, which is referred to as Hall current. Numerous studies show ${ }^{17-20}$ that the Hall current is several times greater than the dis-

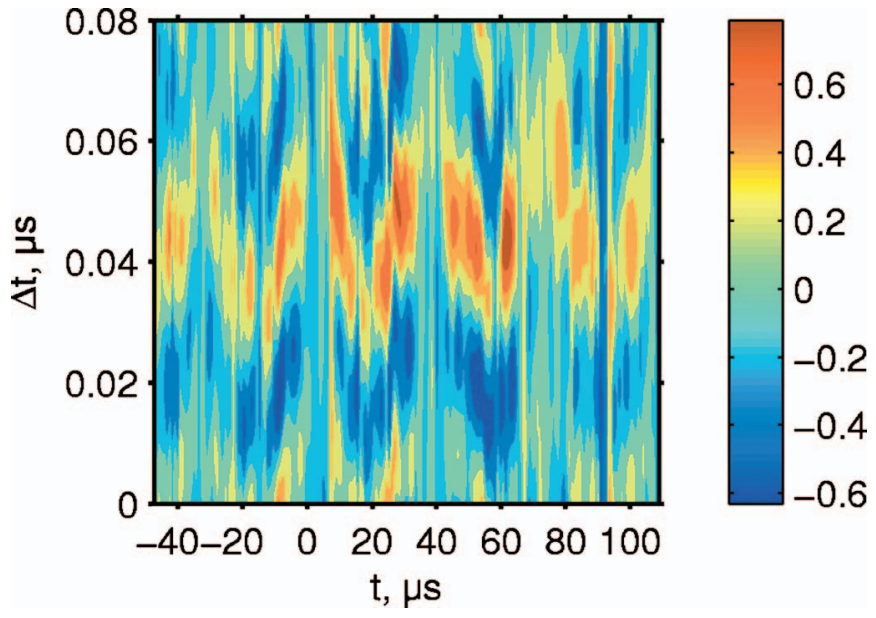

FIG. 8. (Color) Cross-correlation function for the B3 and B7 coils. The offset of the maxima in the cross-correlation (red color) relative to the time lag on the vertical axis indicates a time shift between the signals.

charge current and is also modulated at the LF scale. Therefore, it is reasonable to suggest that the Hall current is a main physical source of signal for axially oriented coils such as B6.

The $100-500 \mathrm{kHz}$ frequency range corresponds to the "transit-time" (TT) oscillations in Hall thrusters, which are usually related to the ion passage through the channel; ${ }^{1,2,12,33}$ some reports extend the upper limit of these oscillations to several MHz. ${ }^{2}$ According to some studies, ${ }^{2}$ the TT instability could be quite intense, but to our knowledge there have been no reports on the significance of oscillations in this frequency range, in Hall thrusters that are currently under development. The $\mathrm{B} 6$ spectrum in this $100-500 \mathrm{kHz}$ frequency range is flat, in contrast to the spectra of B3 and A8 (see Fig. 7). This observation corroborates the hypothesis of ion motion being responsible for TT oscillations. The ion current has a mostly axial direction, thus generating an almost azimuthal magnetic field that is detected by coils B3 and B7.

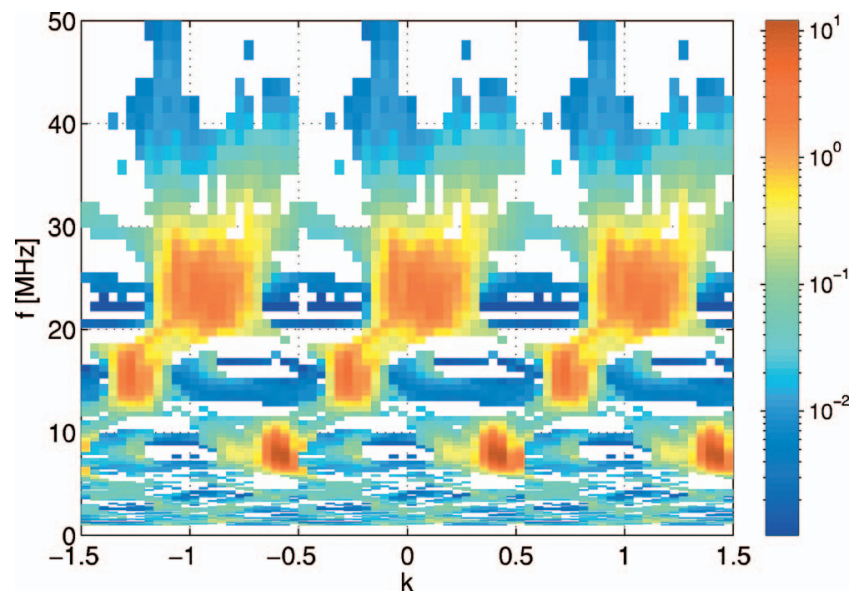

FIG. 9. (Color) Dispersion relation estimated from coils B3 and B7. The vertical scale corresponds to the logarithm of the power spectral density. The periodicity in $k$ is a consequence of the Nyquist limit. The wave number $k$ is normalized with respect to the value corresponding to a dipolar structure. The alignment of the maxima along a line going through $(\omega=0, k=0)$ attests to a dispersionless motion. 
Fluctuations in this frequency range are also present in Langmuir probe spectra, thereby suggesting that various plasma parameters change on this time scale. Taking into account the linear frequency response of coils [see Eq. (3)], we conclude that the TT oscillations are weaker than the LF ones in this thruster operation mode.

Our high-frequency band is limited here at $30 \mathrm{MHz}$ because of the coil resonance and the unstable operation of the electronic components. Frequencies between 5 and $30 \mathrm{MHz}$ correspond to the high-frequency (HF) oscillations that have attracted much attention in the past ${ }^{22,23}$ and again recently. ${ }^{24-27}$ Such HF instabilities generate azimuthal waves that propagate with velocities close to the electron drift velocity $\left(\sim 10^{6} \mathrm{~m} / \mathrm{s}\right)$ in the crossed electric and magnetic fields. ${ }^{22-27}$ The impact of instabilities in the HF frequency range on particle diffusion was recently unambiguously demonstrated in fully kinetic numerical simulations. ${ }^{34}$ Figure 7 shows a strong correlation between the HF spectra of the Langmuir probe and the three coils. The cross-correlation function of B7 and B3 signals, obtained with a rectangular moving window of $2^{10}$ samples with $50 \%$ overlapping, gives a signal time shift on the order of $50 \mathrm{~ns}$ (Fig. 8). This indicates that the source propagates in the azimuthal direction with a velocity of about $2 \times 10^{6} \mathrm{~m} / \mathrm{s}$. The same velocity can be obtained from the autocorrelation functions of each coil and the Langmuir probe. These observations are in perfect agreement with recent studies with the help of antennas and probes. ${ }^{24-27}$ The close properties of coil and probe signals in the time and frequency domains already suggests a common underlying physical process, to be discussed below.

We also analyzed the HF component of the coil signals by making use of wavelets, which are better suited for the study of such a quasistationary wave-field. From this we can estimate the power spectral density versus pulsation $\omega$ and wave number $k{ }^{35} \mathrm{~A}$ linear dispersion relation is found, see Fig. 9. In the same figure, domains where the coherence between the signals is below 0.75 are left in white. From the linear dispersion relation, we conclude that the plasma perturbations rotate azimuthally as a rigid body.

\section{B. Magnetic field}

We deduced the value of induced magnetic field outside of the channel (at the location of the coils) by applying the transfer function obtained from the calibration [see Eq. (6)]. The magnetic field is modulated at the LF scale with an amplitude of about $10^{-5} \mathrm{~T}$ (Fig. 10). This value is several orders of magnitude lower than the stationary magnetic field of the thruster magnetic system: about $0.015 \mathrm{~T}$ in the channel and $0.02 \mathrm{~T}$ at the detecting coils positions. Nevertheless, in the core of the plasma region that stretches from a few $\mathrm{mm}$ inside to a few $\mathrm{mm}$ outside the channel, the induced magnetic field could be significantly higher. A simple $1 / r$ spatial variation, suggested by Eq. (2), leads to rather high induced fields there, at the axial location of coils, assuming a radial location of the field generating current somewhere within the core. This is coherent with other studies reporting a one order of magnitude only lower induced magnetic field near the exit of the channel..$^{20,36}$
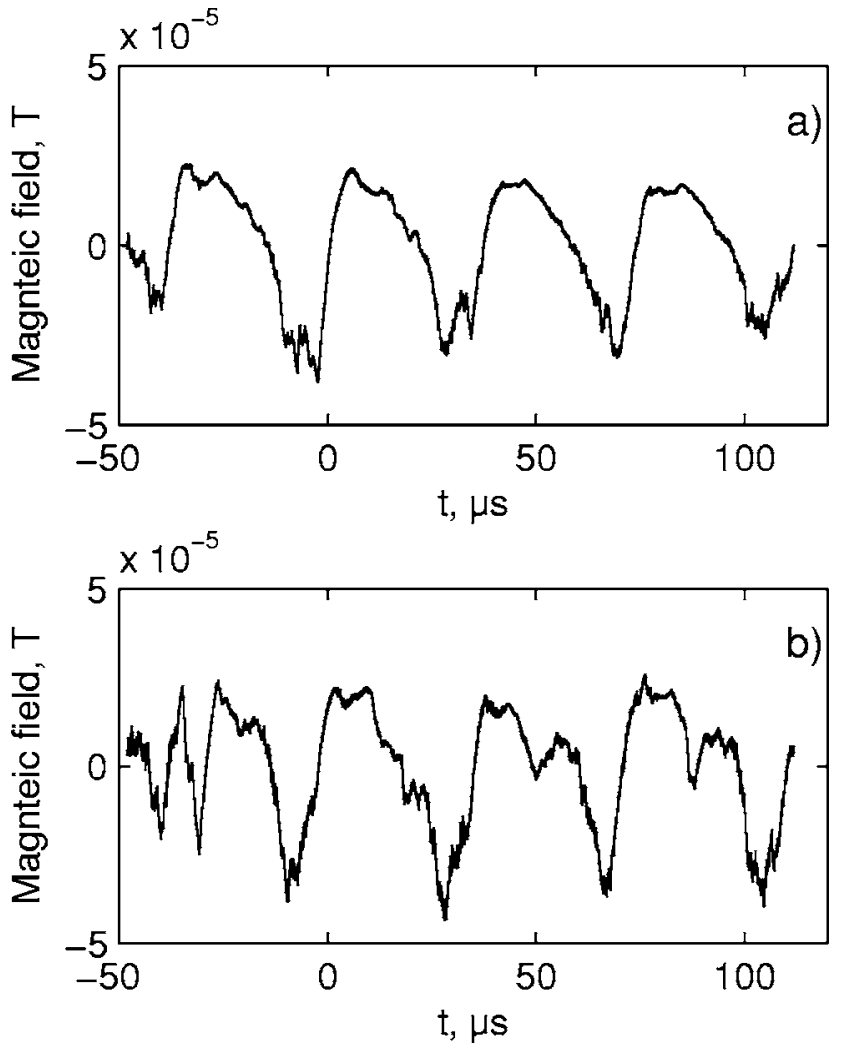

FIG. 10. Magnetic field detected by coils B6 (a) and B7 (b).

The HF component of magnetic field was extracted by applying a digital Butterworth high-pass filter with a cutoff frequency of $2 \mathrm{MHz}$. Some examples of the filtered data are shown in Fig. 11. The HF component of the magnetic field is one order of magnitude weaker than the LF component.

The phase difference between the axial and azimuthal components of the magnetic field is presented in Fig. 12. Notice periodic variations of the phase difference between 1.5-3.5 rad, which are caused by the LF modulation (see Fig. 6). Unfortunately, it is impossible to reconstitute the exact direction of the induced magnetic field without the third $r$ component.

\section{INTERPRETATION}

The amplitude of the induced magnetic field that is measured outside of the channel is three to four orders of magnitude lower than the continuous magnetic field $\left(\sim 10^{-2} \mathrm{~T}\right)$. As we shall see, however, this induced magnetic field is very important for electron diffusion, and allows the electron diffusion coefficients to be evaluated. The locally measured induced magnetic field in addition provides valuable information on the current distributions and thereby on charged particle motions in the Hall thruster. We just saw that the spectral characteristics of the wave-field correspond well to known classes of oscillations in Hall thrusters. The origins of LF $(20-40 \mathrm{kHz})$ and TT $(100-500 \mathrm{kHz})$ phenomena are more or less understood through numerous experimental, theoretical, and numerical studies. ${ }^{1,2,12,31-33}$ A less studied 

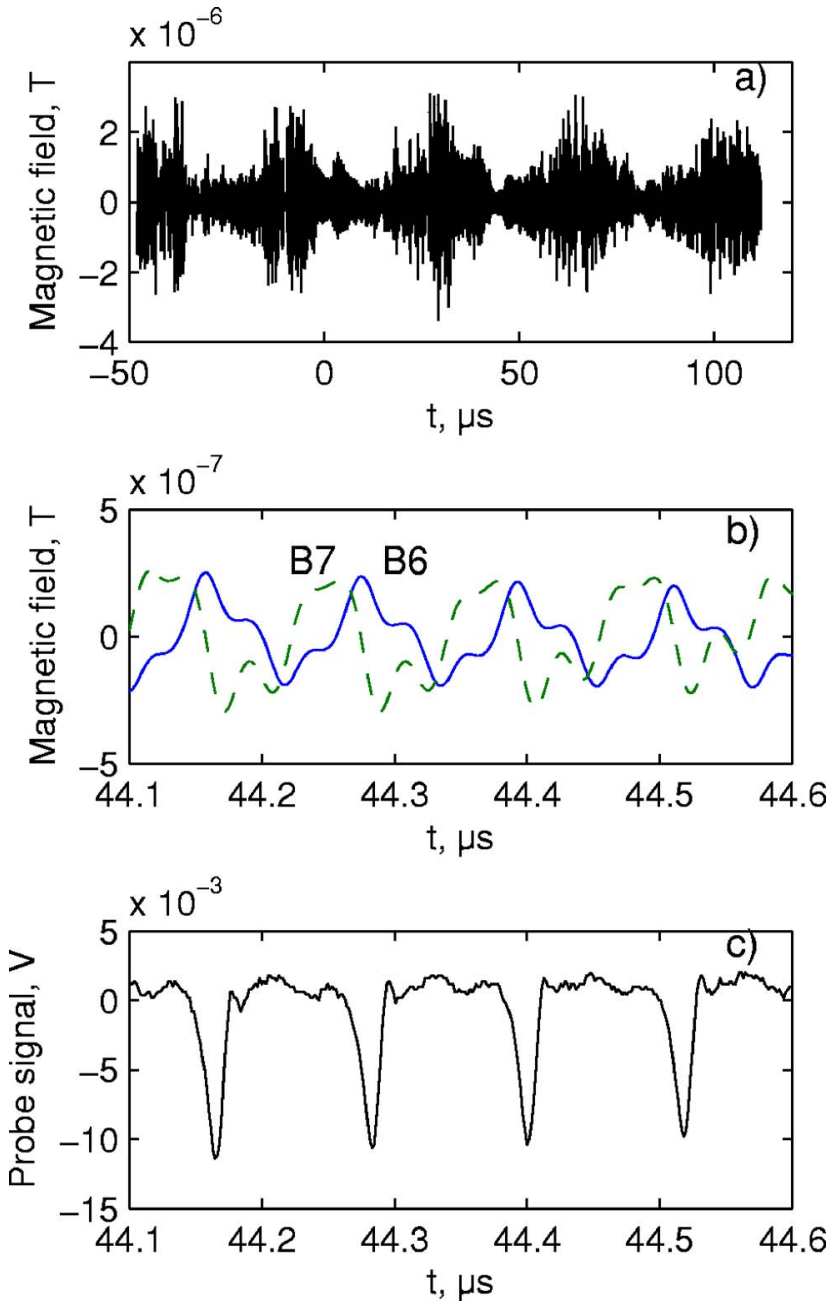

FIG. 11. (Color online) (a) HF component of a magnetic field; (b) excerpt of the coil signal; (c) excerpt of the probe signal.

part of magnetic field spectra concerns HF oscillations above $5 \mathrm{MHz}$, which are limited in our case to $30 \mathrm{MHz}$ by the diagnostics.

We will consider two models in an attempt to understand the generation mechanism of the HF magnetic field. The first of them is related to the hypothesis of local electron density perturbations moving in the azimuthal direction with a velocity close to the electron drift velocity. ${ }^{24,26,27}$ The second is inspired from speculations that the HF instability may induce a sufficient number of anomalous electron collisions for generating a notable perpendicular electron transport (discussed later in this section). Since the HF instability is suggested to be localized in azimuthal and axial directions, ${ }^{26}$ this perpendicular electron transport could be represented by a system of localized electron currents. This instability arises in the plasma volume and it has been found to be the strongest at the exit part and outside of the channel. Therefore, our modeling will deal with the electron transport outside of the channel and that part of the anomalous transport inside the channel that is due to plasma turbulence.

Magnetic fields in plasmas are generated by charged particle currents as described by the Maxwell equation,

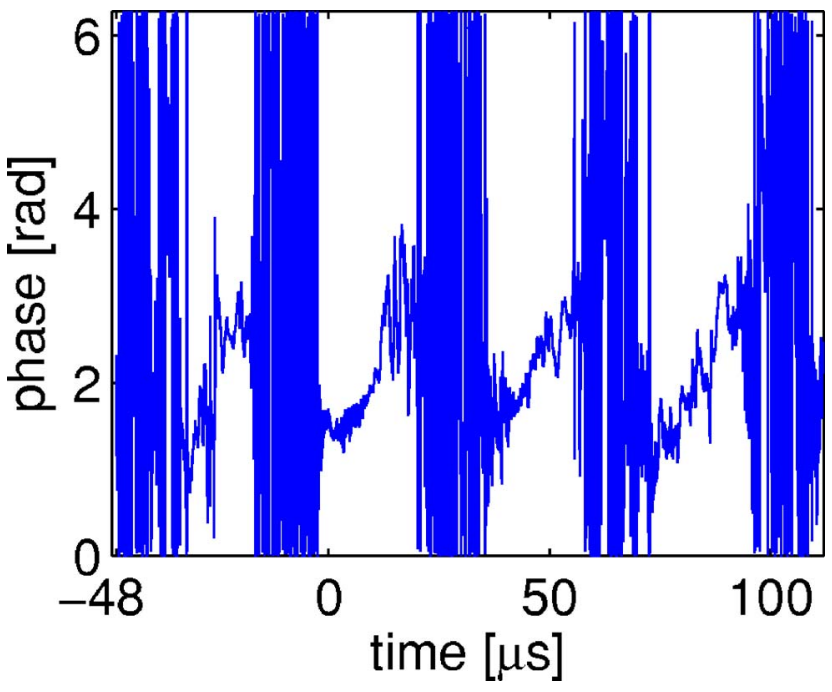

FIG. 12. (Color online) Phase shift between the axial and azimuthal components of the magnetic field (coils B6 and B7). Periods with rapidly changing values coincide with the absence of correlation between signals in the $\mathrm{HF}$ domain (sometimes because of a saturation of the amplifiers) and should therefore be discarded.

$$
\nabla \times \vec{H}=\vec{J}+\frac{\partial \vec{D}}{\partial t},
$$

where $H$ is the magnetic field, $J$ is the charged particle current density, and $D$ is the electric field. In the case of Hall thrusters, the charged particles-electrons and ions-move with velocities well below the speed of light $c$. These velocities could be directly estimated from the characteristic discharge voltage $300 \mathrm{~V}$. In reality, electron characteristic velocities are even smaller because electrons are effectively trapped by magnetic field. Electron drift velocities in crossed electric and magnetic fields are of the order of $10^{6} \mathrm{~m} / \mathrm{s}$ for a magnetic field of $20 \mathrm{mT}$ and an electric field of $10^{4} \mathrm{~V} / \mathrm{m}$. Therefore, particle movement is nonrelativistic and the relativistic effects can be neglected here. We will also neglect displacement currents.

\section{A. Magnetic field from the localized charge}

Let us consider a point charge located on the middle radius of the accelerating channel (in the case of a SPT$100 \mathrm{ML}$ thruster, the radius is $42.5 \mathrm{~mm}$ ) and turning with the pulsation $\omega=2 \pi f$ corresponding to the first HF spectral peak $f=8 \mathrm{MHz}$ (see Fig. 7). The axial propagation velocity of the charge is zero. We simulated the motion of such a charge using Liénard-Wiechert potentials, ${ }^{37}$

$$
\varphi=\frac{1}{4 \pi \varepsilon_{0}} \frac{e}{\left(R-\frac{\vec{v} \vec{R}}{c}\right)}, \quad \vec{A}=\frac{\mu_{0}}{4 \pi} \frac{e \vec{v}}{\left(R-\frac{\vec{v} \vec{R}}{c}\right)},
$$

where $\varphi$ is the electric potential, $\vec{A}$ is the vector potential, $\vec{v}$ is the charge velocity, and $R$ is the distance from the charge to the observation point. The magnetic field $H$ is calculated according to

$$
\vec{H}=\operatorname{rot} \vec{A} \text {. }
$$




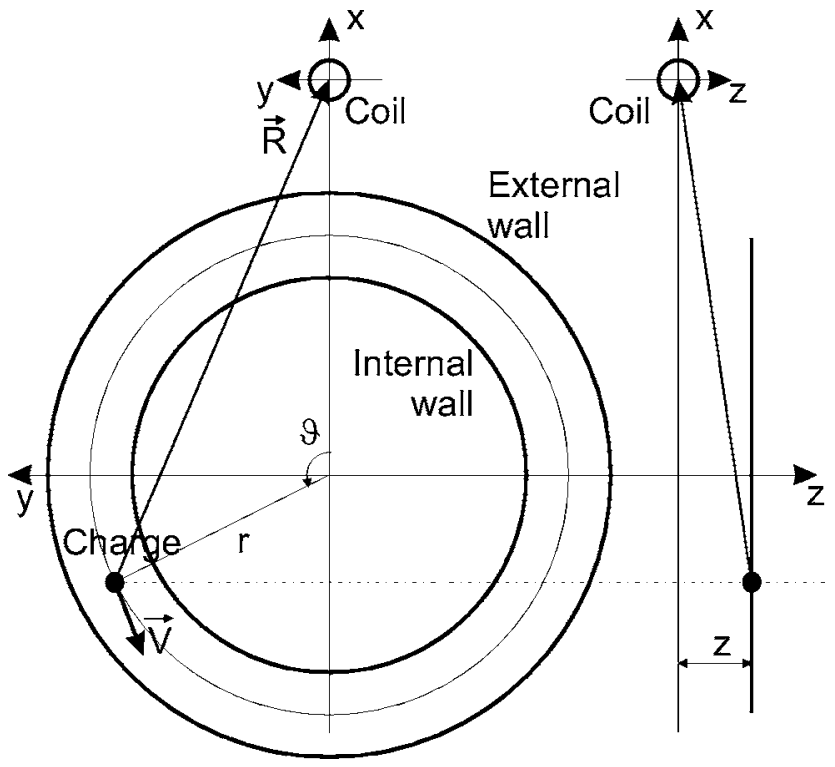

FIG. 13. Scheme of magnetic field calculation from a point charge.

The calculation scheme is presented in Fig. 13. The magnetic field was calculated at the actual location of the detecting coil, i.e., at $R_{c}=70 \mathrm{~mm}$, assuming its axial position is the origin of axial coordinate $(z=0 \mathrm{~mm})$ and coincides with the thruster exit. The value of electric charge was adjusted to obtain the experimental values of magnetic field (see Figs. 10 and 11). With such charges, the calculated azimuthal magnetic field $B_{\theta}$ is much smaller than the axial one $B_{z}$ (Fig. 14). This could be changed with charges moving far away from the detecting coil. The phase relation between the azimuthal and axial magnetic fields, however, does not agree with the observations (see Fig. 12). Furthermore, the axial position of the rotating charge is limited to a few $\mathrm{cm}$ outside of the channel because of the rapidly decreasing electric field (see, for example, Refs. 1, 2, and 5), which leads to the cancellation of azimuthal electron drift. Therefore, the hypothesis of a localized charge moving azimuthally cannot reproduce well the observed picture.

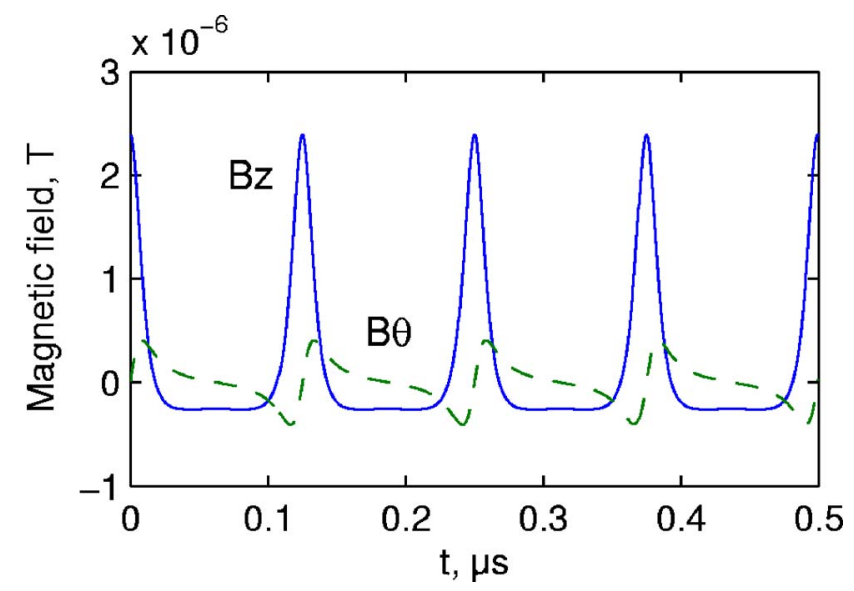

FIG. 14. (Color online) Magnetic field simulated for an azimuthally moving charge.

\section{B. Magnetic field from the localized currents}

Let us now consider in the plasma volume a system of localized currents of the charged particles, modeled by infinitely thin wires of finite length. The currents can be oriented in an arbitrary manner, but the whole current system is supposed to turn around the center of the channel as a rigid body with the frequency $\omega=2 \pi f$ corresponding to the first HF spectral peak $f=8 \mathrm{MHz}$. The contributions to the magnetic field were calculated separately for each current and then added to obtain the resulting field. The calculation was done with the help of the vector potential $\vec{A}$,

$$
\vec{A}=\frac{\mu_{0}}{4 \pi} \int \frac{\vec{I}}{R} d L
$$

where $\vec{I}$ is the current of charged particles, and $d L$ is the infinitely small linear element of the current. The magnetic field is again calculated according to (9) at the location $R_{c}$ $=70 \mathrm{~mm}, z=0 \mathrm{~mm}$, coinciding with the thruster exit plane.

For simplicity, three types of elementary currents are considered: an axially oriented current $I_{z}$, an azimuthal current $I_{\theta}$, and a radial current $I_{r}$. The absolute value of all the currents was chosen to be equal to the estimated electron current through the magnetic barrier, i.e., corresponding to a discharge current of $I_{d}=4.2 \mathrm{~A}$, the values of elementary currents were taken to be $0.2 \times I_{d}$. Azimuthal $B_{\theta}$ and axial $B_{z}$ components of the magnetic field from each elementary current are presented in Figs. 15(a)-15(c). It can be seen that the sole axial current $I_{z}$ [Fig. 15(a)] cannot reproduce the experimentally observed HF magnetic field (see Fig. 11). A closer matching could be obtained by adding azimuthal [Fig. 15(b)] and radial [Fig. 15(c)] currents. Actually, these elementary currents, when placed in the limits of the assumed electron turbulent transport zone $(r=3.5-8 \mathrm{~cm}, z=0-3 \mathrm{~cm})$, defined by the channel dimensions and the relative cathode-channel position, give a correct order of magnitude for the magnetic field. To obtain a correct phase relation, however, these elementary currents should be shifted azimuthally relatively to each other. For example, the resulting magnetic field shown in Fig. 15(d) corresponds to the configuration given in Fig. 16. This configuration gives a phase shift between resulting $z$ and $\theta$ components of about $\pi / 2$, which agrees with the experimentally observations (see Fig. 12). Such a configuration can model a spiral-like current that starts outside the channel close to the cathode radial and axial position and flowing into the channel, at the same time rotating with a velocity close to the electron drift one. In this example, the elementary currents are not connected; their possible connections are shown by dashed lines. Accounting for these connections will of course change the resulting magnetic field. Notice, however, that the localized current approximation is a rather strong one, since the real currents in the plasma volume have a certain distribution and their directions can deviate from being strictly axial, azimuthal, or radial.

A more consistent reconstruction of plasma volume currents could be obtained with a thorough experimental mapping of the turbulent magnetic field, in particular with the help of intrusive probing. This, however, would bring addi- 

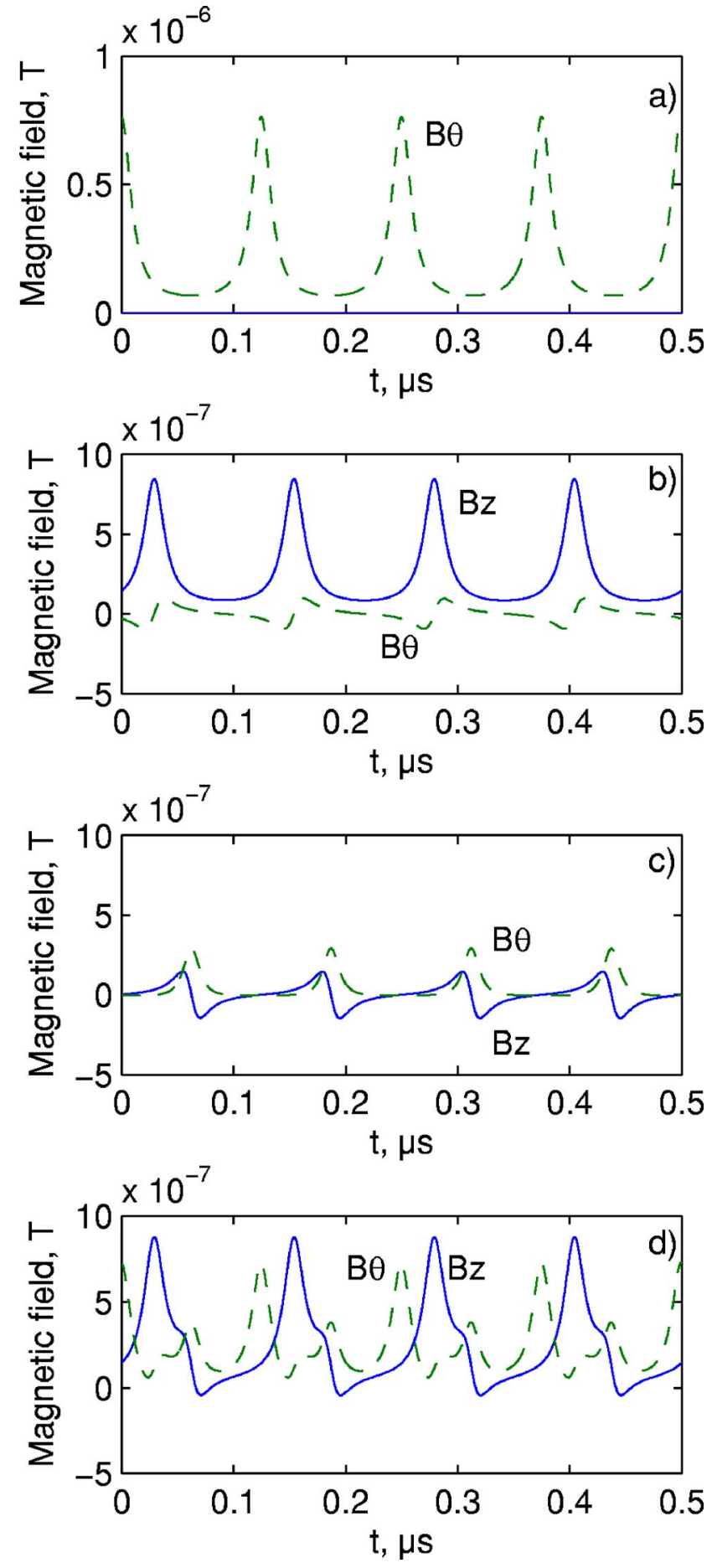

FIG. 15. (Color online) Magnetic field simulated for localized plasma currents: (a) from an axial current, (b) from an azimuthal current, (c) from a radial current, (d) sum of all contributions.

tional perturbations to the discharge. At this point, we propose to consider our model as a phenomenological one, not intending to get the exact current reconstruction. Although this model lacks solid theoretical proof, it agrees quantitatively with the experimental picture. This model is also coherent with the subsequent derivation of the electron "effective" collision frequency.

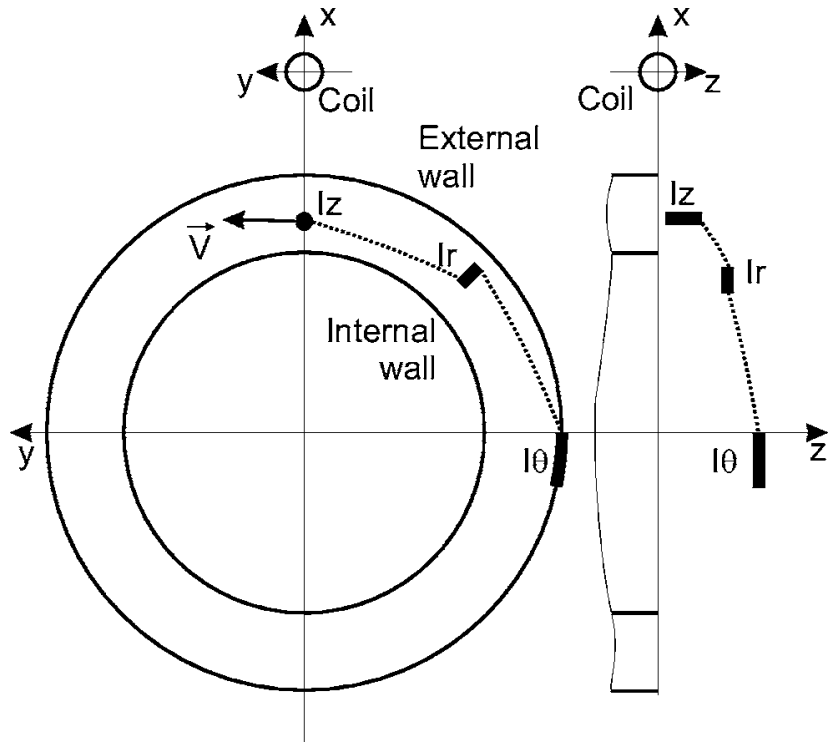

FIG. 16. Configuration of elementary currents, corresponding to Fig. 15; bold, length of the currents for simulation in Fig. 15; dashed, possible connections between the currents; direction of rotation is shown (vector $\mathrm{V}$ ).

\section{Relation to the electron transport}

Theoretical studies ${ }^{23,33,38,39}$ predict the existence of unstable modes in all the above discussed frequency ranges, but according to fully kinetic simulations, ${ }^{34}$ it is an instability in the $\mathrm{MHz}$ range that leads to the generation of the azimuthal turbulent electric field and to the "anomalous" electron transport. Our experiments and the earlier works ${ }^{22-27}$ have confidently identified the azimuthal wave with a fundamental frequency $f \approx 5-10 \mathrm{MHz}$, though with generally low wave numbers $k \sim 20$ to $200 \mathrm{~m}^{-1}$. We will now proceed with the analysis of this instability in order to estimate an "effective" electron collision frequency.

The plasma conditions in the thruster are

$$
\Omega_{e} \ll \omega_{p}, \quad \beta_{e, i}=\mu_{0} \frac{2 k_{B} n T_{e, i}}{B^{2}} \ll 1,
$$

where $\Omega_{e}, \omega_{p}$ are the electron gyro and plasma frequencies, $T_{e} \sim 5 \mathrm{eV}, T_{i} \ll T_{e}$ are, respectively, the electron and ion temperatures, and $n \sim 10^{17} \mathrm{~m}^{-3}$ is the electron density at the exit of the thruster. The high-frequency waves of interest are in the frequency range between electron and ion gyrofrequencies $\Omega_{i} \ll \omega \ll \Omega_{e}$ and are supposed to propagate almost perpendicularly to the ambient magnetic field $k_{\perp} \gg k_{\|}$. Such azimuthal waves belong to so called quasi-electrostatic waves in low hybrid frequency range. Their frequency dependence is described by the following relation: ${ }^{40,41}$

$$
\omega^{2}=\frac{\Omega_{e}^{2} k^{2} c^{2}}{\omega_{p}^{2}\left(1+\frac{k^{2} c^{2}}{\omega_{p}^{2}}\right)}\left[\mu_{m}+\cos ^{2} \theta \frac{k^{2} c^{2}}{\omega_{p}^{2}\left(1+\frac{k^{2} c^{2}}{\omega_{p}^{2}}\right)}\right],
$$

where $\mu_{m}=m_{e} / M_{i}$ is the electron to ion mass ratio and $\theta$ is the angle between the direction of magnetic field and the direction of wave propagation. In these oscillations, the electrons are magnetized while the ions are not, $k_{\perp} \rho_{e} \ll 1$ and $k_{\perp} \rho_{i} \gg 1$, where $\rho_{e}$ and $\rho_{i}$ are, respectively, the electron and 
ion Larmor radia. These waves were shown to be generated in Hall thrusters due to the resistive instability studied by Litvak and Fisch. ${ }^{38}$

These quasi-electrostatic waves are known to play an important role in space plasmas in particle transport and acceleration; they are observed in the polar ionosphere and in the vicinity of quasiperpendicular collisionless shocks, ${ }^{41,42}$ where they are supposed to play an important role in particle acceleration. The particularity of wave particle interaction for these waves consists in the fact that electrons in these motions are magnetized while ions are not. As a consequence, electron motion is mostly affected by the electric field component parallel to the background magnetic field, while ions interact with the major component of the electric field. Because of this, these waves can transfer momentum from parallel electron motion to perpendicular ion motion and vice versa. In the presence of an external electric field, which is the case here, energy can also be transferred. A theoretical study of these waves is beyond the scope of this paper, so we will use known formulas from the literature to evaluate the "effective" collision frequency. This characteristic "effective" or "anomalous" collision frequency can be deduced from direct in situ measurements of magnetic field fluctuations.

The rate of the electron momentum loss due to the generation of waves can be estimated as follows:

$$
\nu_{\text {eff }} m_{e} n V_{d}=-F_{\text {fr }},
$$

where $\nu_{\text {eff }}$ is the "effective" collision frequency, $V_{d}$ is the characteristic drift velocity of electrons, $n$ is their density, and $F_{\mathrm{fr}}$ is the characteristic friction force that appears due to the instability with respect to the generation of waves. This friction force can be evaluated as follows: ${ }^{15,16}$

$$
F_{\text {fr }}=\frac{2}{(2 \pi)^{3}} \int \gamma_{k} W_{k} \frac{k}{\omega_{k}} d^{3} k,
$$

where $\gamma_{k}$ is the increment of the instability due to the electron drift motion that is responsible for the instability generation. Thus this "effective" frequency can be estimated as follows:

$$
\nu_{\text {eff }}=\frac{2}{(2 \pi)^{3} m_{e} n V_{d}^{2}} \int \gamma_{k} W_{k} \frac{k}{\omega_{k}} d^{3} k .
$$

When the electrons are magnetized and ions are not, the macroscopic motions of electrons can be determined by the "effective" diffusion, and ion motion is governed by the macroscopic electric fields. The perpendicular diffusion coefficient for electron motions in this case can be derived from the "effective" collision frequency as $D_{\perp}=\nu_{\text {eff }} \rho_{e}^{2}$ and it can be evaluated if the level of wave activity $W=\int W_{k} d^{3} k$ and the characteristic increment are known. The level of wave activity can be estimated from the wave electric field $W_{k}$ $=\varepsilon_{0} E^{2} / 2$. To estimate the characteristic amplitude of the electric field, we make use of Ampere's law (7), neglecting the displacement current, and taking the estimate of the perpendicular ("anomalous") current density, assuming that it is carried mostly by electrons, $J_{\perp}=-e n V_{e \perp}$ $=-e n\left(\left|\delta \vec{E} \times \vec{B}_{0}\right| / B_{0}^{2}\right) \approx-e n\left(\delta E / B_{0}\right)$, where $\delta E$ is the fluctuat- ing (turbulent) electric field that is assumed to be perpendicular to the stationary magnetic field $B_{0}$. From (7) we have $|\nabla \times \delta \vec{H}|=\left|\left(1 / \mu_{0}\right) \nabla \times \delta \vec{B}\right| \approx\left(1 / \mu_{0}\right) k \delta B$, where $\delta B$ is the turbulent magnetic field and $k$ is the wave number. This leads to the following relation between the fluctuating magnetic and electric fields:

$$
\delta E \approx \frac{\Omega_{e}}{\omega_{p}^{2}} \frac{k}{\varepsilon_{0} \mu_{0}} \delta B,
$$

where $k$ is the wave number of the experimentally detected azimuthal HF wave. In the simplest case, its wavelength is equal to the channel circumference $2 \pi R$, but as was demonstrated in Refs. 26 and 27, these HF waves can have much smaller wavelengths (down to at least $1 \mathrm{~cm}$ ), which leads to $k \sim(0.2-6.3) \times 10^{2} \mathrm{~m}^{-1}$. From this we get $\delta E$ $\sim 0.14-4.4 \mathrm{~V} / \mathrm{cm}$, and thus $W_{k} / m_{e} n V_{d}^{2} \approx W_{k} / k_{B} n T_{e}$ $\approx \varepsilon_{0} \delta E^{2} / 2 k_{B} n T_{e} \sim 10^{-5}-10^{-3}$. It is rather difficult to evaluate the increment of the instability, but for rather strong deviations from equilibrium one can take it to be of the order of $\gamma_{k} \approx 0.1 \times \omega_{\mathrm{LH}}=0.1 \times \Omega_{e}\left(m_{e} / M_{i}\right)^{1 / 2} \approx 5 \times 10^{5} \mathrm{~s}^{-1}$. In this case, the characteristic "anomalous" collision frequency will be of the order of $5 \times 10^{2} \mathrm{~s}^{-1}$. This value is significantly smaller than "classical" collision frequency. However, this evaluation utilized the oscillation intensities measured outside the region of denser plasma in front of the channel (cf. coil positions in Fig. 5). Taking the calculated dependencies of the magnetic field, one finds that the field amplitude, while approaching the core of this region, can increase by as much as 10 to 30 . The effective collision frequency can therefore reach values of the order of $\left(5 \times 10^{4}\right)-(1$ $\left.\times 10^{6}\right) \mathrm{s}^{-1}$. From this, the turbulent electric field can reach approximately $10^{2} \mathrm{~V} / \mathrm{cm}$, which is comparable to the axial electric field. The possibility of generating such strong turbulent electric fields was demonstrated numerically. ${ }^{34}$ This can result in a quite strong effect as the characteristic "collisional" frequency in the same region of the thruster is evaluated to be of the order of $\nu=10^{5} \mathrm{~s}^{-1}$. We conclude that in the vicinity of the channel, the diffusive behavior of the electrons is governed by turbulence, whereas in the periphery of the flow, classical collisions with neutrals prevail.

\section{CONCLUSIONS}

The turbulent magnetic field measurements in a Hall thruster reveal the nature of the physical processes that govern electron transport. Although the turbulent magnetic field that is measured outside of the channel is too weak to affect charged particles directly, it provides relevant information on their dynamics. The characteristics of the magnetic field oscillations we observe in the low-frequency and transit-time frequency ranges are in a good agreement with properties derived from other types of diagnostics. Magnetic field measurements give complementary information about ion acceleration processes and Hall current evolution.

The basic hypothesis underlying the interpretation of the nonstationary magnetic field measurements is the generation of this field by moving charged particles in the plasma 
volume. We have shown that the source of HF instabilities can be represented by localized currents with different orientations. This model is in good agreement with the experimental results. More detailed studies with all three components of the magnetic field in principle allow the current distribution to be reconstructed.

The key result of this study is the first direct experimental evaluation of the "effective" or "anomalous" collision frequency due to the plasma turbulence in Hall thrusters. It has been shown that in the central region just outside the thruster, the electron dynamics is determined by "effective" or "anomalous" collisions associated with the plasma turbulence. In the periphery, however, classical collisions are more important. The effective collision frequency is found to be sufficiently smaller than the one used in computer simulations (see, for example, Refs. 8 and 9). This "low effectiveness" of anomalous transport processes may be one of the reasons for the strongly nonstationary dynamics of the thruster.

We have thereby demonstrated that the localized (in axial, radial, and azimuthal directions) detection of the nonstationary magnetic fields represents a valuable and very important type of diagnostic for probing nonstationary processes in Hall thrusters. Such a diagnostic can be both nonintrusive and intrusive.

\section{ACKNOWLEDGMENTS}

The authors acknowledge the technical support of $\mathrm{P}$. Dom and the PIVOINE team: S. Sayamath, C. Legentil, and P. Lasgorceix. This work was performed in the frame of the research group GDR n²759 CNRS/CNES/SNECMA/ Universities "Propulsion Spatiale à Plasma."

${ }^{1}$ A. I. Morozov and V. V. Savelyev, Rev. Plasma Phys. 21, 203 (2000).

${ }^{2}$ V. V. Zhurin, H. R. Kaufman, and R. S. Robinson, Plasma Sources Sci. Technol. 8, R1 (1999).

${ }^{3}$ H. Gray, S. Provost, M. Glogowski, and A. Demaire, in Proceedings of the 29th International Electric Propulsion Conference, Princeton, NJ (Electric Rocket Propulsion Society, Worthington, 2005), IEPC-05-082.

${ }^{4}$ C. R. Koppel and D. Estublier, in Proceedings of the 29th International Electric Propulsion Conference, Princeton, NJ (Electric Rocket Propulsion Society, Worthington, 2005), IEPC-05-119.

${ }^{5}$ V. Kim, J. Propul. Power 14, 736 (1998).

${ }^{6}$ K. Komurasaki and Y. Arakawa, J. Propul. Power 11, 1317 (1995).

${ }^{7}$ J. M. Fife, Ph.D. thesis, Massachusetts Institute of Technology (1998).

${ }^{8}$ G. J. M. Hagelaar, J. Bareilles, L. Garrigues, and J.-P. Boeuf, J. Appl. Phys. 91, 5592 (2002).

${ }^{9}$ G. J. M. Hagelaar, J. Bareilles, L. Garrigues, and J.-P. Boeuf, J. Appl. Phys. 93, 67 (2003).

${ }^{10}$ J. W. Koo and I. D. Boyd, Phys. Plasmas 13, 033501 (2006).
${ }^{11}$ M. K. Scharfe, N. Gascon, M. A. Capelli, and E. Fernandez, Phys. Plasmas 13, 083505 (2006).

${ }^{12}$ E. Y. Choueiri, Phys. Plasmas 8, 1411 (2001).

${ }^{13}$ G. S. Janes and R. S. Lowder, Phys. Fluids 9, 1115 (1966).

${ }^{14}$ N. B. Meezan, W. A. Hargus, Jr., and M. A. Cappelli, Phys. Rev. E 63, 026410 (2001).

${ }^{15}$ A. A. Galeev and R. Z. Sagdeev, Nonlinear Plasma Theory, in Reviews of Plasma Physics Vol. 7, edited by M. A. Leontovich (Consultant Bureau, New York, 1979), pp. 1-180 (translated from Russian).

${ }^{16}$ A. A. Galeev and R. Z. Sagdeev, Current Instabilities and Anomalous Resistivity of Plasma, in Handbook of Plasma Physics, Basic Plasma Physics, edited by A. A. Galeev and R. N. Sudan (North Holland, Amsterdam, 1984), Vol. 2, pp. 272-303.

${ }^{17}$ A. Bouchoule, Ch Philippe-Kadles, M. Prioul et al., Plasma Sources Sci. Technol. 10, 364 (2001).

${ }^{18}$ V. N. Dem'yanenko, I. P. Zubkov, S. V. Lebedev, and A. I. Morozov, Sov. Phys. Tech. Phys. 23, 376 (1978).

${ }^{19}$ A. I. Bugrova, V. S. Versotskii, and V. K. Kharchevnikov, Sov. Phys. Tech. Phys. 25, 1307 (1980).

${ }^{20}$ C. A. Thomas, N. Gascon, and M. A. Capelli, Phys. Rev. E 74, 056402 (2006).

${ }^{21}$ D. C. Black, R. M. Mayo, and R. W. Caress, Phys. Plasmas 4, 3581 (1997).

${ }^{22}$ G. G. Shishkin and V. F. Gerasimov, Zh. Tekh. Fiz. XLV, 1847 (1975).

${ }^{23}$ Y. V. Esipchuck and G. N. Tilinin, Sov. Phys. Tech. Phys. 21, 417 (1976).

${ }^{24}$ M. Prioul, Ph.D. thesis, Orleans University, France (2002).

${ }^{25}$ A. A. Litvak, Y. Raitses, and N. J. Fisch, Phys. Plasmas 11, 1701 (2004).

${ }^{26}$ A. Lazurenko, V. Vial, M. Prioul, and A. Bouchoule, Phys. Plasmas 12, 013501 (2005).

${ }^{27}$ A. Lazurenko, L. Albarède, and A. Bouchoule, Phys. Plasmas 13, 083503 (2006).

${ }^{28}$ M. Parrot, D. Benoist, J. J. Berthelier et al., Planet. Space Sci. 54, 456 (2006).

${ }^{29}$ C. Cavoit, Rev. Sci. Instrum. 77, 064703 (2006).

${ }^{30}$ A. Bouchoule, A. Cadiou, A. Heron, M. Dudeck, and M. Lyszyk, Contrib. Plasma Phys. 41, 573 (2001).

${ }^{31}$ N. Yamamoto, K. Komurasaki, and Y. Arakawa, J. Propul. Power 21, 870 (1998).

${ }^{32}$ J.-P. Bœuf and L. Garrigues, J. Appl. Phys. 84, 3541 (1998).

${ }^{33}$ S. Barral, K. Makowski, Z. Peradzynski, and M. Dudeck, Phys. Plasmas 12, 073504 (2005).

${ }^{34}$ J. C. Adam, A. Héron, and G. Laval, Phys. Plasmas 11, 295 (2004).

${ }^{35}$ T. Dudok de Wit, V. V. Krasnoselskikh, S. D. Bale, M. W. Dunlop, H. Lühr, S. J. Schwartz, and L. J. C. Woolliscroft, Geophys. Res. Lett. 22, 2653 (1995).

${ }^{36}$ P. Y. Peterson, A. D. Gallimore, and J. M. Haas, Phys. Plasmas 9, 4354 (2002).

${ }^{37}$ L. D. Landau and E. M. Lifshitz, The Theory of Field, 7th ed. (Nauka, Moscow, 1988), p. 216.

${ }^{38}$ A. A. Litvak and N. J. Fisch, Phys. Plasmas 8, 648 (2001).

${ }^{39}$ A. A. Litvak and N. J. Fisch, Phys. Plasmas 11, 1379 (2004).

${ }^{40}$ A. B. Mikhailovskii, Instabilities of a Homogeneous Plasma, Theory of Plasma Instabilities Vol. 1 (Consultant Bureau, New York, 1974) (translation from Russian).

${ }^{41}$ V. Krasnoselskikh, E. Kruchina, G. Thejappa, and A. Volokitin, Astron. Astrophys. 149, 323 (1985).

${ }^{42}$ O. Vaisberg, A. Galeev, G. Zastenker, S. Klimov, M. Nozdrachev, R. Sagdeev, A. Sokolov, and V. Shapiro, Sov. Phys. JETP 58, 716 (1983). 